\title{
Multiple-gap response of type-I noncentrosymmetric BeAu superconductor
}

\author{
Rustem Khasanov (®), ${ }^{1, *}$ Ritu Gupta $\odot,{ }^{1}$ Debarchan Das $\odot,{ }^{1}$ Alfred Amon, ${ }^{2}$ Andreas Leithe-Jasper, ${ }^{2}$ and Eteri Svanidze ${ }^{2}$ \\ ${ }^{1}$ Laboratory for Muon Spin Spectroscopy, Paul Scherrer Institute, 5232 Villigen, Switzerland \\ ${ }^{2}$ Max-Planck-Institut für Chemische Physik fester Stoffe, Nöthnitzer Straße 40, 01187 Dresden, Germany
}

(Received 19 December 2019; revised manuscript received 25 March 2020; accepted 7 April 2020; published 8 May 2020)

\begin{abstract}
Precise measurements of the thermodynamic critical field $\left(B_{\mathrm{c}}\right)$ in the type-I noncentrosymmetric superconductor $\mathrm{BeAu}$ were performed by means of the muon-spin rotation and relaxation technique. The temperature evolution of $B_{\mathrm{c}}$ cannot be described within the single-gap scenario and it requires the presence of at least two different types of superconducting order parameters. The self-consistent two-gap approach, adapted for analysis of $B_{\mathrm{c}}(T)$ behavior, suggests the presence of two superconducting energy gaps with the gap-to- $T_{\mathrm{c}}$ ratios $2 \Delta / k_{\mathrm{B}} T_{\mathrm{c}} \simeq 4.52$ and $\simeq 2.37$ for the big gap and the small gap, respectively $[\Delta=\Delta(T=0)$ is the zero-temperature value of the gap and $k_{\mathrm{B}}$ is the Boltzmann constant]. This implies that the superconductivity in $\mathrm{BeAu}$ is unconventional and that the supercarrier pairing occurs at various energy bands.
\end{abstract}

DOI: 10.1103/PhysRevResearch.2.023142

\section{INTRODUCTION}

$\mathrm{BeAu}$ is an old known superconductor with a transition temperature $T_{\mathrm{c}} \simeq 3.2 \mathrm{~K}$. Superconductivity in BeAu was originally discovered by Matthias in 1959 [1], i.e., just two years after the formulation of BCS theory [2]. In this short report, Matthias noted the absence of superconductivity in pure $\mathrm{Be}$ and $\mathrm{Au}$ (Be was later found to have $T_{\mathrm{c}} \simeq 26 \mathrm{mK}$ [3]) and performed a search within the gold-rich site of the Be-Au phase diagram. The superconductivity was found to appear in a stoichiometric (i.e., 1:1 Be-to-Au ratio) BeAu sample [1].

Recently, the interest in $\mathrm{BeAu}$ was renewed [4-8]. This mostly relates to the realization of its noncentrosymmetric crystal structure, which was expected to give rise to unconventional superconductivity due to spin-orbit coupling and/or mixed singlet-triplet pairing state (see, e.g., Refs. [9-16] and references therein). In addition, the $B 20 \mathrm{FeSi}$ type of crystal structure of BeAu becomes particularly interesting since such materials were predicted to host chiral fermions in topological semimetals [17-19]. Moreover, B20 structure is the only known crystal structure for bulk magnetic skyrmions in materials such as $\mathrm{MnSi}, \mathrm{Fe}_{1-x} \mathrm{Co}_{x} \mathrm{Si}$, FeGe, $\mathrm{MnGe}, \mathrm{Cu}_{2} \mathrm{OSeO}_{3}$, etc. [20-24]. All these make $\mathrm{BeAu}$ an intriguing candidate material to search for unconventional superconductivity, associated with its noncentrosymmetric crystal structure in combination with the possible existence of exotic quasiparticles.

The previously published papers agreed that $\mathrm{BeAu}$ is characterized by an isotropic superconducting energy gap and, predominantly, by an $s$-wave spin-singlet pairing in the weak-

\footnotetext{
*rustem.khasanov@psi.ch

Published by the American Physical Society under the terms of the Creative Commons Attribution 4.0 International license. Further distribution of this work must maintain attribution to the author(s) and the published article's title, journal citation, and DOI.
}

coupling regime [4-8]. There is, however, disagreement on the type of superconductivity. An intensive characterization of BeAu by means of the specific heat, dc magnetization, ac susceptibility, and resistivity performed by Rebar et al. [4,6] revealed a crossover from type-I to type-II superconductivity at approximately $1.2 \mathrm{~K}$, thus putting $\mathrm{BeAu}$ in the class of so-called type-1.5 or type-II/I superconductors [25,26]. On the other hand, purely type-I behavior was reported by Singh et al. [7] and Beare et al. [8] based on the results of dc magnetization, specific heat, and muon-spin rotation and relaxation experiments. In addition, the results of de Haas-van Alphen experiments combined with density functional theory (DFT) band-structure calculations reveal the presence of multiple (at least three) conductive bands crossing the Fermi level $[4,6]$. Consequently, if superconductivity in $\mathrm{BeAu}$ sets in different energy bands, the effects of a multiple-band structure are expected to influence the supercarrier formation. This is, e.g., the case for the famous two-gap superconductor $\mathrm{MgB}_{2}$, the broad variety of Fe-based and cuprate high-temperature superconductors, etc. (see, e.g., Refs. [27-42] and references therein). Note, however, that until now the multiple-band features were not detected on the measured thermodynamical quantities and the superconductivity of $\mathrm{BeAu}$ was treated within the single-band approach [4-8].

In this paper we report on the results of the precise measurements of the thermodynamical critical field $B_{\mathrm{c}}$ of $\mathrm{BeAu}$ by means of the muon-spin rotation and relaxation $(\mu \mathrm{SR})$ technique. The analysis of $B_{\mathrm{c}}(T)$ within the self-consistent two-gap approach reveals the presence of two superconducting energy gaps with the ratios $2 \Delta / k_{\mathrm{B}} T_{\mathrm{c}} \simeq 4.52$ and $\simeq 2.37$ for the big and the small gap, respectively $[\Delta=\Delta(T=0)$ is the zero-temperature value of the gap and $k_{\mathrm{B}}$ is the Boltzmann constant]. Our results imply that the multiple-band superconductivity can also be realized in type-I superconducting materials, in analogy with that reported in Refs. [43,44].

The paper is organized as follows. Section II describes the $\mathrm{BeAu}$ sample and the experimental measurement procedure. 

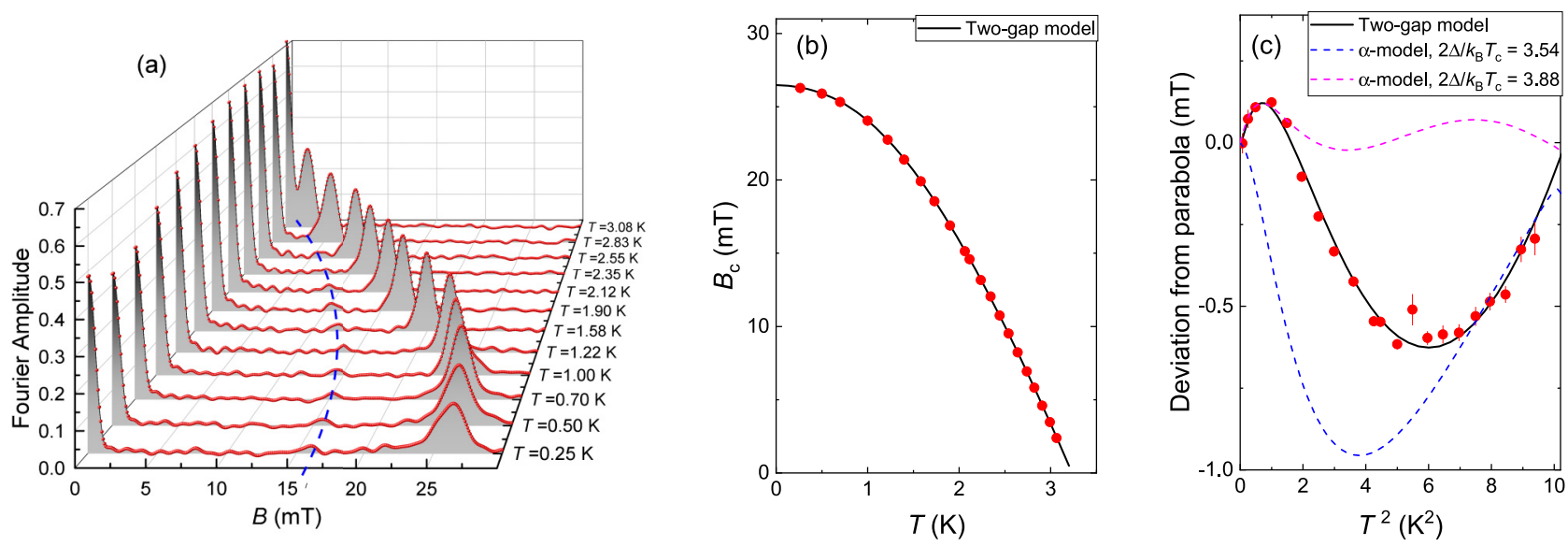

FIG. 1. (a) Fast Fourier transform of the TF- $\mu$ SR time spectra of BeAu for several $T$ and $B_{\text {ex }}$ values. The dashed blue line represents the position of the external field $B_{\mathrm{ex}}$. Temperatures are marked on the $y$ axis. The tiny background peak at the $B=B_{\mathrm{ex}}$ position corresponds to the muons which missed the sample. (b) The temperature evolution of the thermodynamical critical field of BeAu obtained from the $B>B_{\text {ex }}$ peak position. The solid line is the fit of the self-consistent two-gap model [Eq. (6)] to the experimental $B_{\mathrm{c}}(T)$. (c) The deviation of $B_{\mathrm{c}}(T)$ from parabolic dependence. The dashed blue and pink lines are expectations for the single $s$-wave gap. The solid black line is the self-consistent two-gap fit. See text for details.

Results of the thermodynamic critical field measurements by means of the muon-spin rotation and relaxation technique are summarized in Sec. III. The analysis of the temperature evolution of $B_{\mathrm{c}}$ within the single- and double-gap scenario is given in Sec. IV. The summary and conclusions follow in Sec. V. The Appendixes describe the data analysis procedure and the results of the fit of the single-gap $\alpha$ model to the experimental data.

\section{SAMPLE PREPARATION AND EXPERIMENTAL TECHNIQUES}

The polycrystalline $\mathrm{BeAu}$ samples were the same as used by Beare et al. in Ref. [8]. The muon-spin rotation and relaxation measurements were performed at the $\pi \mathrm{E} 1$ beamline by using the Dolly spectrometer (Paul Scherrer Institute, PSI Villigen, Switzerland). The ${ }^{4} \mathrm{He}$ cryostat equipped with the ${ }^{3} \mathrm{He}$ inset was used. Samples were sandwiched between two pieces of a thin copper foil ( $\simeq 10 \mu \mathrm{m}$ each), which are transparent to positive surface muons used in our studies. The foil/sample assembly was screwed to the frame-shaped sample holder and attached, via $\simeq 1$-cm-diam-thick copper rod, to the bottom part of the ${ }^{3} \mathrm{He}$ inset. Such construction, on the one side, allows to ensure a good thermal contact between the sample and the ${ }^{3} \mathrm{He}$ pot and, on the other side, permits the use of a "veto" mode of the $\mu$ SR spectrometer. The veto mode rejects the muons missing the sample and, as a consequence, reduces the background of the $\mu$ SR response to almost zero [see tiny background peaks following the dashed blue line in Fig. 1(a) and Ref. [45] for the detailed explanations of the veto mode principle]. The external magnetic field $B_{\mathrm{ex}}$ was applied parallel to the direction of the muon momentum and perpendicular to the initial muon spin direction, which corresponds to the transverse field (TF) $\mu$ SR geometry. Several elliptically shaped disks of BeAu $(0.5 \mathrm{~mm}$ thick and roughly $3.0 \times 4.0 \mathrm{~mm}^{2}$ in size) were used. The field was applied perpendicular to the flat faces of the samples. The experiments were performed in the temperature range $0.25-5 \mathrm{~K}$ and in the field range of 0.5 to $50 \mathrm{mT}$ (see Appendix A for details).

\section{EXPERIMENTAL RESULTS}

The TF- $\mu$ SR experiments were performed in the intermediate state of the BeAu superconducting sample, i.e., when the sample volume is separated on the normal state and the superconducting (Meissner) state domains [3,7,8,46-53]. The $B$ - $T$-scan measuring scheme, as discussed by Karl et al. in Ref. [52], was used. Each measured point was reached in two steps: first by stabilizing the temperature and second by swiping the field to $B>B_{\mathrm{c}}$ (up to $30 \mathrm{mT}$ in our case) and decreasing it back to the measuring one. The idea of such a scheme is twofold: (i) to keep unchanged the volume parts of the sample occupied by the normal state $\left(f_{\mathrm{N}}\right)$ and the superconducting state $\left(f_{\mathrm{S}}\right)$ domains $(f$ denotes the volume fraction) and (ii) to maintain similarly distributed domain patterns for each $B-T$ measuring point. Note that the shapes of the domain patterns in type-I superconductors depend strongly on the magnetic history $[49,50]$. The $B-T$ points were taken along the $\simeq 0.6 B_{\mathrm{c}}(T)$ line by considering the $B_{\mathrm{c}}(T)$ curve as is determined in Refs. [7,8] (see Appendix A for details).

The magnetic field distributions in the BeAu sample, as obtained from the Fourier transform of TF- $\mu$ SR time spectra, are shown in Fig. 1(a). The dashed blue line represents the position of the external field $B_{\mathrm{ex}}$. Down to the lowest temperature studied $(T \simeq 0.25 \mathrm{~K})$, the signal splits into two peaks positioned at $B=0$ and $B>B_{\mathrm{ex}}$, respectively. Such distributions are typical for type-I superconducting materials in the intermediate state [51-53]. Our results, therefore, confirm the conclusion of Refs. [7,8] on type-I superconductivity of $\mathrm{BeAu}$. The area below the peaks corresponds to the superconducting (Meissner) state $\left(f_{\mathrm{S}}\right)$ and the normal state $\left(f_{\mathrm{N}}\right)$ volume fractions, while the position of the $B>B_{\text {ex }}$ peak determines the value of the thermodynamic critical field $B_{\mathrm{c}}$ [51-53]. The temperature evolution of $B_{\mathrm{c}}$, obtained from the fit of TF- $\mu \mathrm{SR}$ 
data, is shown in Fig. 1(b). The details of the data analysis procedure, as well as the temperature dependencies of the fitting parameters, are presented in Appendix A.

\section{DISCUSSIONS}

The deviation of the $B_{\mathrm{c}}$ vs $T$ curve from the parabolic function $D\left(T^{2}\right)=B_{\mathrm{c}}\left(T^{2}\right)-B_{\mathrm{c}}(0)\left[1-\left(T / T_{\mathrm{c}}\right)^{2}\right]$ is shown in Fig. 1(c). Following Refs. [54,55] the shape of the $D\left(T^{2}\right)$ function depends strongly on the $2 \Delta / k_{\mathrm{B}} T_{\mathrm{c}}$ ratio and it is also expected to be sensitive to the symmetry of the superconducting energy gap.

Bearing in mind the isotropic single-gap behavior reported in Refs. [4,6], the initial analysis of the $B_{\mathrm{c}}(T)$ dependence of BeAu was performed by means of the phenomenological $\alpha$ model within the single $s$-wave gap approach [54,55]. The fit procedure and the results obtained from such analysis are described in Appendix B. The analysis reveals that the $D\left(T^{2}\right)$ data cannot be described within the single-gap scenario. The low- and the high-temperature parts of $D\left(T^{2}\right)$ require rather different $2 \Delta / k_{\mathrm{B}} T_{\mathrm{c}}$ values. To put this statement into evidence, the dashed pink and blue lines in Fig. 1(c) correspond to the theoretical $D\left(T^{2}\right)$ curves with $2 \Delta / k_{\mathrm{B}} T_{\mathrm{c}}=3.88$ and 3.54 , respectively. The disagreement between the single $s$-wave gap analysis and the experimental data implies, therefore, that in BeAu a more complicated gap scenario needs to be considered.

By taking into account the presence of multiple bands crossing the Fermi level, as is reported in de Haas-van Alphen experiments $[4,6]$, as well as confirmed by DFT calculations [4-6], the further analysis of the temperature evolution of the thermodynamic critical field was performed within the self-consistent two $s$-wave gap scenario.

Following Refs. [56-58], within the two-gap scenario the coupled $s$-wave gap equations are described as

$$
\begin{aligned}
\Delta_{1}(T)= & \int_{0}^{\omega_{D}} \frac{n_{1} V_{11} \Delta_{1}(T)}{\sqrt{E^{2}+\Delta_{1}^{2}(T)}} \tanh \frac{\sqrt{E^{2}+\Delta_{1}^{2}(T)}}{2 k_{B} T} d E \\
& +\int_{0}^{\omega_{D}} \frac{n_{2} V_{12} \Delta_{2}(T)}{\sqrt{E^{2}+\Delta_{2}^{2}(T)}} \tanh \frac{\sqrt{E^{2}+\Delta_{2}^{2}(T)}}{2 k_{B} T} d E, \\
\Delta_{2}(T)= & \int_{0}^{\omega_{D}} \frac{n_{1} V_{21} \Delta_{1}(T)}{\sqrt{E^{2}+\Delta_{1}^{2}(T)}} \tanh \frac{\sqrt{E^{2}+\Delta_{1}^{2}(T)}}{2 k_{B} T} d E \\
& +\int_{0}^{\omega_{D}} \frac{n_{2} V_{22} \Delta_{2}(T)}{\sqrt{E^{2}+\Delta_{2}^{2}(T)}} \tanh \frac{\sqrt{E^{2}+\Delta_{2}^{2}(T)}}{2 k_{B} T} d E .
\end{aligned}
$$

Here $\Delta_{1}(T)$ and $\Delta_{2}(T)$ are temperature evolutions of gaps within bands 1 and $2 ; n_{1}$ and $n_{2}$ are the partial densities of states for each band at the Fermi level $\left(n_{1}+n_{2}=1\right) ; V_{11}\left(V_{22}\right)$ and $V_{12}\left(V_{21}\right)$ are the intraband and the interband interaction potentials, respectively. For simplicity, it is also assumed that the Debye frequency $\left(\omega_{\mathrm{D}}\right)$ is the same for both bands.
The thermodynamic critical field $B_{\mathrm{c}}$ was determined from the normal state $\left(F_{\mathrm{N}}\right)$ and the superconducting state $\left(F_{\mathrm{S}}\right)$ free energy difference [46]:

$$
\frac{B_{\mathrm{c}}^{2}(T)}{8 \pi}=F_{\mathrm{N}}(T)-F_{\mathrm{S}}(T) .
$$

Considering the presence of two superconducting energy bands, the second term on the right-hand side of the above equation consists of two contributions:

$$
F_{\mathrm{S}}(T)=F_{\mathrm{S}, 1}\left[T, \Delta_{1}(T)\right]+F_{\mathrm{S}, 2}\left[T, \Delta_{2}(T)\right] .
$$

Following Johnston [55], the individual free energy components are further derived as

$$
\frac{F_{\mathrm{N}}(T)}{\gamma_{\mathrm{e}} T_{\mathrm{c}}^{2}}=-\frac{T^{2}}{2 T_{\mathrm{c}}^{2}}
$$

and

$$
\begin{aligned}
\frac{F_{\mathrm{S}, i}\left[T, \Delta_{i}(T)\right]}{\gamma_{\mathrm{e}, i} T_{\mathrm{c}}^{2}}= & -\frac{3}{\pi^{2} k_{\mathrm{B}}^{2} T_{\mathrm{c}}^{2}}\left[\frac{\Delta_{i}(T)^{2}}{4}\right. \\
& \left.+\int_{0}^{\infty} f(E, T) \frac{2 \varepsilon^{2}+\Delta_{i}(T)^{2}}{E} d \varepsilon\right]
\end{aligned}
$$

Here, $i(i=1,2)$ is the band index, $f(E, T)=$ $\left[\exp \left(E / k_{\mathrm{B}} T\right)+1\right]^{-1}$ is the Fermi function, $E=$ $E[\varepsilon, \Delta(T)]=\sqrt{\varepsilon^{2}+\Delta(T)^{2}}$ is the quasiparticle energy, and $\gamma_{\mathrm{e}}\left(\gamma_{\mathrm{e}, i}\right)$ is the total (the $i$ th band) normal state electronic specific heat coefficient. Note that $\gamma_{\mathrm{e}}=\gamma_{\mathrm{e}, 1}+\gamma_{\mathrm{e}, 2}$ and that Eqs. (2), (4), and (5) are expressed in cgs units [55].

Finally, the equation used to fit to the experimental $B_{\mathrm{c}}(T)$ data presented in Fig. 1(b) took the form

$$
\frac{B_{\mathrm{c}}^{2}(T)}{B_{\mathrm{c}}^{2}(0)}=\frac{F_{N}(T)-F_{\mathrm{S}, 1}\left[T, \Delta_{1}(T)\right]-F_{\mathrm{S}, 2}\left[T, \Delta_{2}(T)\right]}{-F_{\mathrm{S}, 1}\left(0, \Delta_{1}\right)-F_{\mathrm{S}, 2}\left(0, \Delta_{2}\right)} .
$$

Here, $\Delta_{1}=\Delta_{1}(T=0)$ and $\Delta_{2}=\Delta_{2}(T=0)$ are the zerotemperature values of the superconducting energy gaps. Note that within the free electron approximation the product $\gamma_{\mathrm{e}, i} / \gamma_{\mathrm{e}}$ entering Eq. (6) is equal to the corresponding partial density of states $n_{i}$ in Eq. (1), so that $\gamma_{\mathrm{e}, 1} / \gamma_{\mathrm{e}} \equiv n_{1}$ and $\gamma_{\mathrm{e}, 2} / \gamma_{\mathrm{e}} \equiv n_{2}$.

The fit requires solving two coupled nonlinear equations [Eq. (1)]. We used MATHCAD 15.0 with the LevenbergMarquardt nonlinear equation solver [59]. The results of the analysis, with the Debye frequency $\omega_{\mathrm{D}} \simeq 25.4 \mathrm{meV}$ [6], are shown by black solid lines in Figs. 1(b) and 1(c). Obviously, the self-consistent two-gap model describes the experimental data remarkably well. The fit parameters are $B_{\mathrm{c}}(0) \simeq 26.47 \mathrm{mT}, n_{1} \simeq 0.65\left(n_{2}=1-n_{1} \simeq 0.35\right), V_{11} \simeq$ $0.246, V_{22} \simeq 0.343, V_{12} \simeq 0.321$, and $V_{21} \simeq 0.0727$. The value of the superconducting transition temperature, corresponding to $B_{\mathrm{c}}=0$, was found to be $T_{\mathrm{c}} \simeq 3.232 \mathrm{~K}$. The temperature evolutions of the big and small gaps and the comparison of their temperature dependencies with the weakcoupled BCS prediction [60] are presented in Fig. 2.

From the analysis of $B_{\mathrm{c}}(T)$ of BeAu within the two-gap approach, the following important points emerge:

(i) The partial density of states for the band(s), where the big superconducting energy gap opens, is almost twice as high as that for the band(s) with the small gap: $n_{1} \simeq 2 n_{2}$. The results of the band-structure calculation, presented in Ref. [5], reveal that the partial density of states at the Fermi 

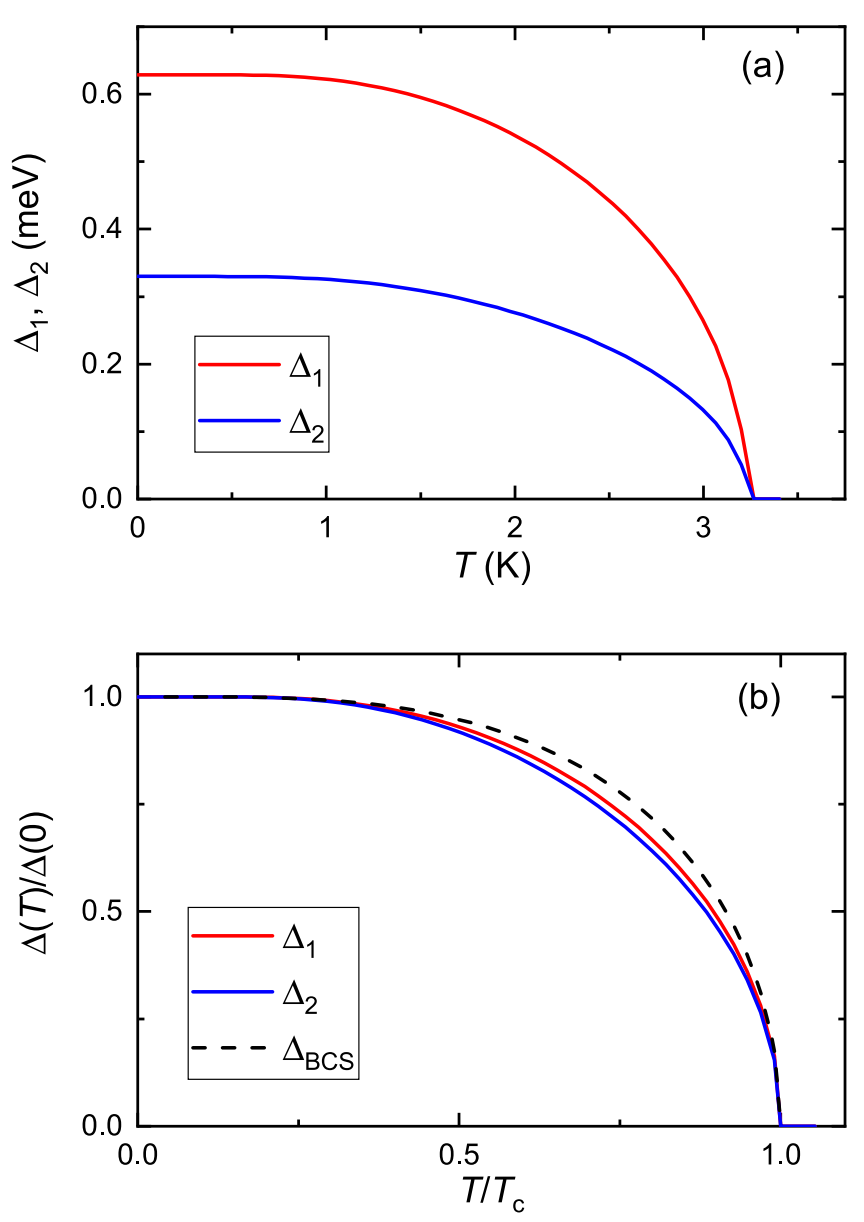

FIG. 2. (a) Temperature evolutions of the big $\left(\Delta_{1}\right)$ and small $\left(\Delta_{2}\right)$ superconducting gaps obtained within the framework of the self-consistent two-gap model. (b) Comparison of temperature dependencies of $\Delta_{1}$ and $\Delta_{2}$ with the weak-coupled BCS prediction [60].

level is dominated by $\mathrm{Be} 2 p$, Au $5 p$, and Au $6 p$ bands, so that $n_{\mathrm{Au} 5 p} \simeq n_{\mathrm{Au} 6 p} \simeq(0.6-0.7) n_{\mathrm{Be} 2 p}$. This suggests that the big gap may be opened in Be $2 p$ and one of the Au bands ( $5 p$ or $6 p)$, while the other Au band $(6 p$ or $5 p)$ carries the small superconducting gap.

(ii) The zero-temperature values of the big and small gaps were estimated to be $\Delta_{1} \simeq 0.62 \mathrm{meV}$ and $\Delta_{2} \simeq 0.32 \mathrm{meV}$. The corresponding gap-to- $T_{\mathrm{c}}$ ratios are $2 \Delta_{1} / k_{\mathrm{B}} T_{\mathrm{c}} \simeq 4.52$ and $2 \Delta_{2} / k_{\mathrm{B}} T_{\mathrm{c}} \simeq 2.37$. Such values suggests strong coupling within the first and weak coupling within the second band, respectively.

(iii) The interband coupling potentials $V_{12} \simeq 0.321$ and $V_{21} \simeq 0.0727$ have relatively high values. In particular, $V_{12}$ is comparable to $V_{22}$ and is even higher than $V_{11}$. This suggests strong coupling between the bands, which also results in almost identical temperature dependencies of the big $\left[\Delta_{1}(T)\right]$ and small $\left[\Delta_{2}(T)\right]$ gaps [see Fig. 2(b)]. Note that in the case of $V_{12}, V_{21} \ll V_{11}, V_{22}, \Delta_{1}(T) / \Delta_{1}$ and $\Delta_{2}(T) / \Delta_{2}$ are very much different $[58,61,62]$.

(iv) The temperature dependencies of both gaps, presented in Fig. 2(b), are weaker than the weak-coupled BCS prediction.
We believe that such effects are related to the BeAu system rather than to the model itself. The lack of absence of the experimental data for BeAu does not allow to make a comparison of $\Delta(T)^{\prime}$ s dependencies obtained in the present study with the directly measured gap values. Note, however, that the recent work of Kim et al. [63] on $\mathrm{MgB}_{2}$, where such data become accessible, reveal that $\Delta(T)$ s obtained within the framework of the self-consistent model match rather well temperature evolutions of superconducting energy gaps measured in tunneling experiments. More interesting is that the "weakening" of the gaps in $\mathrm{MgB}_{2}$ is also observed both experimentally and theoretically.

It is worth noting here that, due to the noncentrosymmetric crystal structure of $\mathrm{BeAu}$, the phase of the superconducting order parameter may change between the different energy bands. In relation to the two $s$-wave gap behavior reported here, both $s^{++}$and $s^{+-}$scenarios could be realized in $\mathrm{BeAu}$. The above-presented self-consistent model is, however, "phase insensitive" and does not allow to distinguish between them. An additional check could be performed by studying the influence of nonmagnetic impurities on the superconducting transition temperature. As shown in Refs. [64,65], the transition temperature $T_{\mathrm{c}}$ in $s^{+-}$superconductors is quickly suppressed due to the pair breaking promoted by interband impurity scattering, while it remains only marginally affected for $s^{++}$materials. A series of $T_{\mathrm{c}}$ and $B_{c}(T)$ measurements of BeAu samples with a different amount of nonmagnetic impurities could be highly reliable and may help to clarify this question.

\section{SUMMARY AND CONCLUSIONS}

To summarize, the precise measurements of the thermodynamic critical field $B_{\mathrm{c}}$ in the noncentrosymmetric $\mathrm{BeAu}$ superconductor were performed by means of the muon-spin rotation and relaxation technique. The main results are the following:

(i) Within the full temperature range studied, the superconductivity in BeAu remains of type-I. No indications of type-II superconductivity for $T \lesssim 1.2 \mathrm{~K}$ and at least down to $T \simeq 0.25 \mathrm{~K}$ were detected.

(ii) The value of the thermodynamic critical field at $T=0$ and the transition temperature $T_{\mathrm{c}}$ were found to be $B_{\mathrm{c}}(0) \simeq$ $26.47 \mathrm{mT}$ and $T_{\mathrm{c}} \simeq 3.232 \mathrm{~K}$, in agreement with the previously published data $[7,8]$.

(iii) The single $s$-wave gap approach does not allow to describe the temperature evolution of the thermodynamic critical field $B_{\mathrm{c}}$, thus calling for the presence of an additional superconducting energy gap(s).

(iv) The self-consistent two-gap model, adapted for analysis of $B_{\mathrm{c}}(T)$ behavior, was developed. It takes into account the intraband coupling within each individual band, as well as the interband coupling between the bands. The model was found to describe accurately the experimental $B_{\mathrm{c}}(T)$ data of BeAu.

(v) The gap-to- $T_{\mathrm{c}}$ ratios were estimated to be $2 \Delta_{1} / k_{\mathrm{B}} T_{\mathrm{c}} \simeq$ 4.52 and $2 \Delta_{2} / k_{\mathrm{B}} T_{\mathrm{c}} \simeq 2.37$. This implies that the strong and weak coupling occur within the first and the second band, respectively.

To conclude, our results suggest that the superconductivity in $\mathrm{BeAu}$ is unconventional and that the supercarrier pairing 


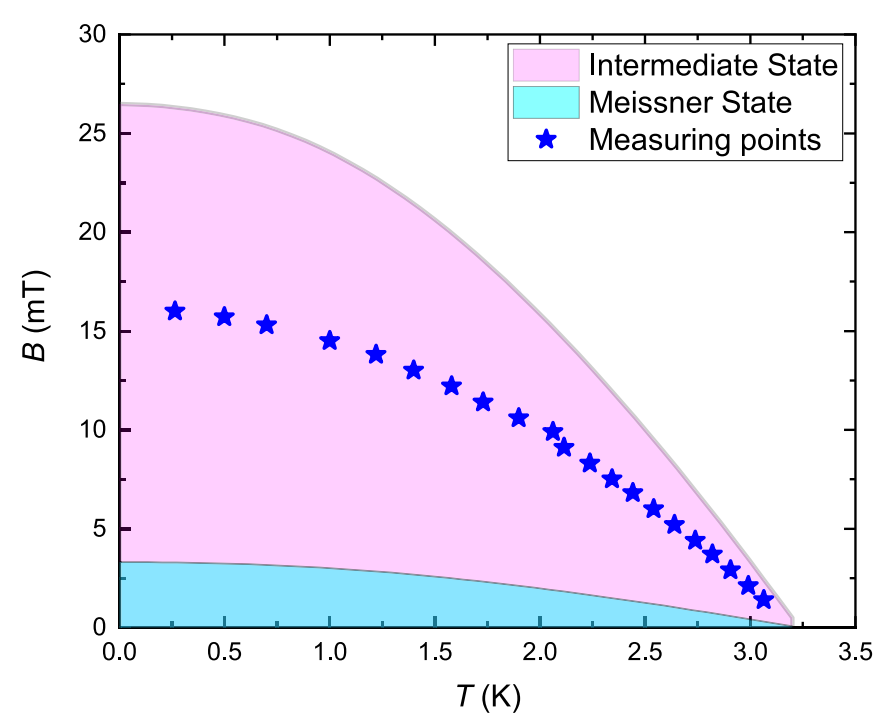

FIG. 3. Phase diagram of superconducting BeAu samples used in present studies. The intermediate state and the Meissner state areas are denoted by pink and cyan, respectively. Blue stars represent the measured $B-T$ points. The phase diagram was determined for a zero-temperature value of the thermodynamic critical field $B_{\mathrm{c}}(0)=$ $26 \mathrm{mT}$, a transition temperature $T_{\mathrm{c}}=3.25 \mathrm{~K}$, and a demagnetization factor $N=0.875$, assuming that the temperature evolution of $B_{\mathrm{c}}$ follows $B_{\mathrm{c}}(T)=B_{\mathrm{c}}(0)\left[1-\left(T / T_{\mathrm{c}}\right)^{2}\right][3,46]$.

takes place in different energy bands. More important, our results imply that the multiple-gap behavior is also realized in type-I superconducting materials.

\section{ACKNOWLEDGMENTS}

This work was performed at the Swiss Muon Source $(\mathrm{S} \mu \mathrm{S})$, Paul Scherrer Institute (PSI, Switzerland). The work of R.G. is supported by the Swiss National Science Foundation (SNF Grant No. 200021-175935). The authors acknowledge the technical support of Toni Shiroka and Chris Baines. Juri Grin is acknowledged for his steady support. R.K. acknowledges helpful discussions with Zurab Guguchia.

\section{APPENDIX A: MUON-SPIN ROTATION AND RELAXATION EXPERIMENTS}

Transverse-field muon-spin rotation and relaxation (TF$\mu \mathrm{SR})$ experiments were carried out at the $\pi \mathrm{E} 1$ beamline by using the dedicated Dolly spectrometer (Paul Scherrer Institute, Switzerland). The surface muons with a momentum $\simeq 28 \mathrm{MeV} / \mathrm{c}$ were used. The sample was cooled down by using an Oxford Sorption Pumped ${ }^{3} \mathrm{He}$ Cryostat (base temperature $\simeq 0.25 \mathrm{~K}$ ). The typical counting statistics was $\sim 5 \times 10^{6}$ positron events for each data point. The experimental data were analyzed using the MUSRFIT package [66].

\section{Measurement procedure}

Experiments were performed by following the " $B-T$-scan" measuring scheme [52]. As described in the main text, the $B-T$ measured points (blue stars in Fig. 3) were approached in two steps: (1) by stabilizing the temperature and (2) by swiping the field to $B>B_{\mathrm{c}}$ and decreasing it back to the measuring one. The idea was to follow the $B-T$ path of BeAu's phase diagram allowing to keep constant volume parts of the sample occupied by the normal state and the Meissner state domains. The $B-T$ points were taken along the $\simeq 0.6 B_{\mathrm{c}}(T)$ line, i.e., in between the $(1-N) B_{\mathrm{c}}(T)$ and $B_{\mathrm{c}}(T)$ curves $(N$ is the demagnetization factor). Note that these lines determine the region of existence of the intermediate state of type-I superconducting materials $[3,46]$.

The boundary between the Meissner and the intermediate state $\left[(1-N) B_{\mathrm{c}}(T)\right.$; see Fig. 3] was determined by using the value of the demagnetization factor $N$ for a circular disk in a perpendicular field [67]:

$$
N^{-1}=1+1.6 \frac{h}{d}
$$

Here $h$ and $d$ denote the height and the diameter of the disk. For elliptically shaped disks of BeAu samples used in present studies $\left(0.5 \mathrm{~mm}\right.$ thick and roughly $3.0 \times 4.0 \mathrm{~mm}^{2}$ in size $)$, $N \simeq 0.875$ is obtained.

\section{Data analysis procedure}

The experimental data were analyzed by separating the $\mathrm{TF}-\mu \mathrm{SR}$ response of the sample (s) and the background (bg) contributions:

$$
A_{0} P(t)=A_{\mathrm{s}} P_{\mathrm{s}}(t)+A_{\mathrm{bg}} P_{\mathrm{bg}}(t) .
$$

$A_{0}$ is the initial asymmetry of the muon-spin ensemble. $A_{\mathrm{s}}$ $\left(A_{\mathrm{bg}}\right)$ and $P_{\mathrm{s}}(t)\left[P_{\mathrm{bg}}(t)\right]$ are the asymmetry and the time evolution of the muon-spin polarization of the sample (background), respectively.

The sample contribution was described by assuming a separation between normal state $(\mathrm{N})$ and superconducting $(\mathrm{S})$ domains:

$$
\begin{aligned}
P_{\mathrm{S}}(t)= & f_{\mathrm{N}} e^{-\lambda_{\mathrm{N}} t} \cos \left(\gamma_{\mu} B_{\mathrm{N}} t+\phi\right) \\
& +f_{\mathrm{S}}\left[\frac{1}{3}+\frac{2}{3}\left(1-\sigma_{\mathrm{GKT}}^{2} t^{2}\right) e^{-\sigma_{\mathrm{GKT}}^{2} t^{2} / 2}\right] .
\end{aligned}
$$

Here, the first term on the right-hand side of the equation corresponds to the sample's normal state response: $f_{\mathrm{N}}$ is the normal state volume fraction $\left(f_{\mathrm{N}}=1\right.$ for $\left.T \geqslant T_{\mathrm{c}}\right), \lambda_{\mathrm{N}}$ is the exponential relaxation rate, and $B_{\mathrm{N}}$ is the internal field $\left[B_{\mathrm{N}}=B_{\mathrm{c}}\right.$ for $T<T_{\mathrm{c}}\left(B_{\mathrm{ex}}\right)$ and $B_{\mathrm{N}}=B_{\mathrm{ex}}$ for $T \geqslant T_{\mathrm{c}}\left(B_{\mathrm{ex}}\right)$, respectively]. The second term describes the contribution of the superconducting part of the sample remaining in the Meissner state $\left(B_{\mathrm{S}}=0\right) . f_{\mathrm{S}}=1-f_{\mathrm{N}}$ is the superconducting volume fraction. The term is approximated by the Gaussian Kubo-Toyabe function with the relaxation rate $\sigma_{\mathrm{GKT}}$, which is generally used to describe the nuclear magnetic moment contribution in zero-field experiments (see, e.g., Ref. [68] and references therein).

The background contribution was described as

$$
P_{\mathrm{bg}}(t)=e^{-\lambda_{\mathrm{bg}} t} \cos \left(\gamma_{\mu} B_{\mathrm{ex}} t+\phi\right),
$$

where $\lambda_{\mathrm{bg}}$ is the exponential relaxation rate, $B_{\mathrm{ex}}$ is the applied field, and $\phi$ is the initial phase of the muon-spin ensemble. The background contribution accounts for the muons stopped outside the sample (in the sample holder and/or in the cryostat's windows and radiation shields). Within the full set of 


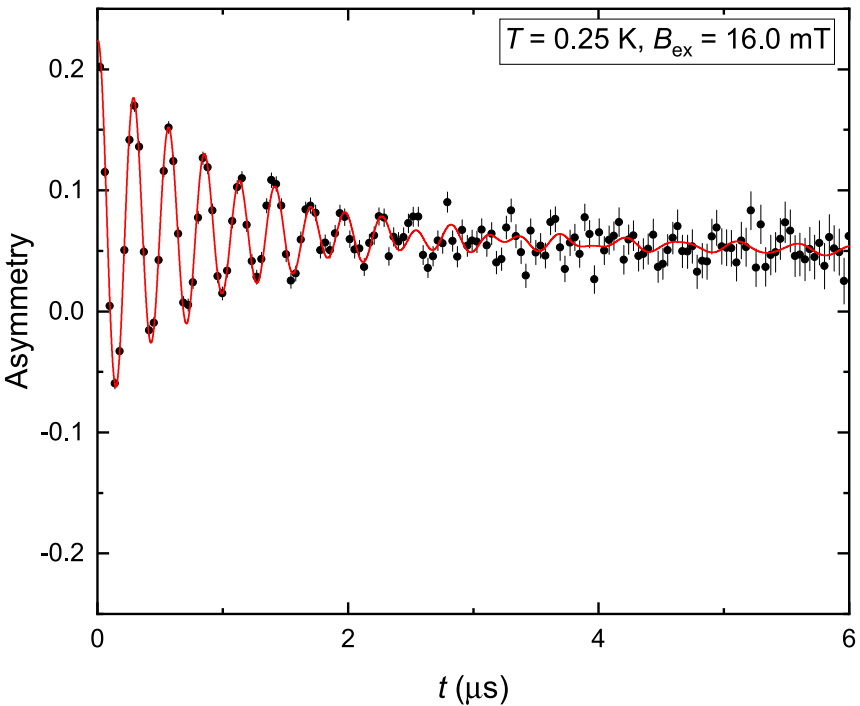

FIG. 4. TF- $\mu$ SR time spectra taken at $B_{\text {ex }}=16 \mathrm{mT}$ and $T \simeq$ $0.25 \mathrm{~K}$. The red line is a fit of Eq. (A2) [with the background and the sample contributions described by Eqs. (A4) and (A3)] to the experimental data.

experiments the background asymmetry $A_{\text {bg }}$ did not exceed $\simeq 3 \%$ of the initial asymmetry $A_{0}$ [see tiny peaks following the external field in Fig. 1(a) in the main text].

The fit of Eq. (A2) to the data, with the background and the sample contributions described by Eqs. (A4) and (A3), was performed globally. All TF- $\mu$ SR time spectra were fitted simultaneously with $A_{\mathrm{s}}, A_{\mathrm{bg}}, \sigma_{\mathrm{GKT}}$, and $\lambda_{\mathrm{bg}}$ as common parameters, and $f_{\mathrm{N}}, B_{\mathrm{N}}, B_{\mathrm{ex}}$, and $\lambda_{\mathrm{N}}$ as individual parameters for each particular data set. Figure 4 shows TF- $\mu$ SR time spectra taken at $B_{\mathrm{ex}}=16 \mathrm{mT}$ and $T \simeq 0.25 \mathrm{~K}$. Figure 5 represents the temperature evolution of the normal state volume fraction $f_{\mathrm{N}}$ [Fig. 5(a)] and the exponential relaxation within the normal state domains $\lambda_{\mathrm{N}}$ [Fig. 5(b)].

\section{APPENDIX B: ANALYSIS OF $B_{\mathrm{c}}(T)$ WITHIN THE SINGLE-GAP $\alpha$ MODEL}

The single-gap $\alpha$ model assumes that the temperature dependence of the normalized superconducting energy gap $[54,55]$,

$$
\delta_{\mathrm{BCS}}(T)=\frac{\Delta(T)}{\Delta}=\frac{\Delta_{\mathrm{BCS}}(T)}{\Delta_{\mathrm{BCS}}},
$$

is the same as in BCS theory [60], and it is calculated for the BCS value $\alpha_{\mathrm{BCS}}=\Delta_{\mathrm{BCS}} / k_{\mathrm{B}} T_{\mathrm{c}} \simeq 1.764$. The $\alpha$ model allows $\alpha=\Delta / k_{\mathrm{B}} T_{\mathrm{c}}$ to be an adjustable parameter [54,55].

The single-gap fit of Eq. (6) to the experimental $B_{\mathrm{c}}(T)$ data with the normal and superconducting state free energy components described by Eqs. (4) and (5) results in $T_{\mathrm{c}}=$ $3.195 \mathrm{~K}, B_{\mathrm{c}}(0)=26.65 \mathrm{mT}$, and $2 \alpha=2 \Delta / k_{\mathrm{B}} T_{\mathrm{c}}=3.78$. The deviation of experimental $B_{\mathrm{c}}(T)$ data from the parabolic dependence $\left[D\left(T^{2}\right)=B_{\mathrm{c}}\left(T^{2}\right)-B_{\mathrm{c}}(0)\left\{1-\left(T / T_{\mathrm{c}}\right)^{2}\right\}\right]$ and their comparison with the result of the $\alpha$-model fit are shown in Fig. 6. Obviously, there is a poor agreement between the experimental data and the fit. This suggests that more
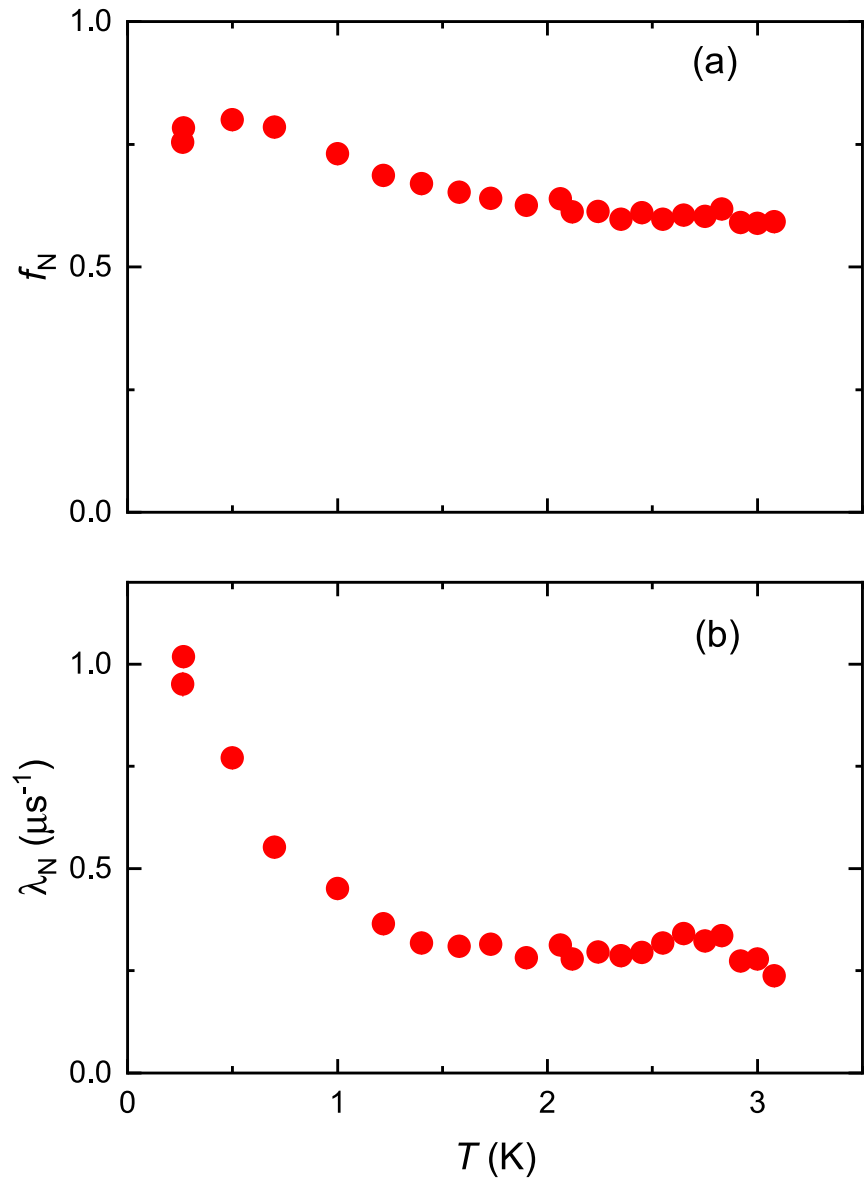

FIG. 5. (a) Dependence of the volume fraction of the normal state domains $f_{\mathrm{N}}$ on temperature. (b) Dependence of the exponential relaxation for normal state domains $\lambda_{\mathrm{N}}$ on temperature.

complicated gap structure, compared to the isotropic single $s$-wave gap, is going to be realized in BeAu superconductors.

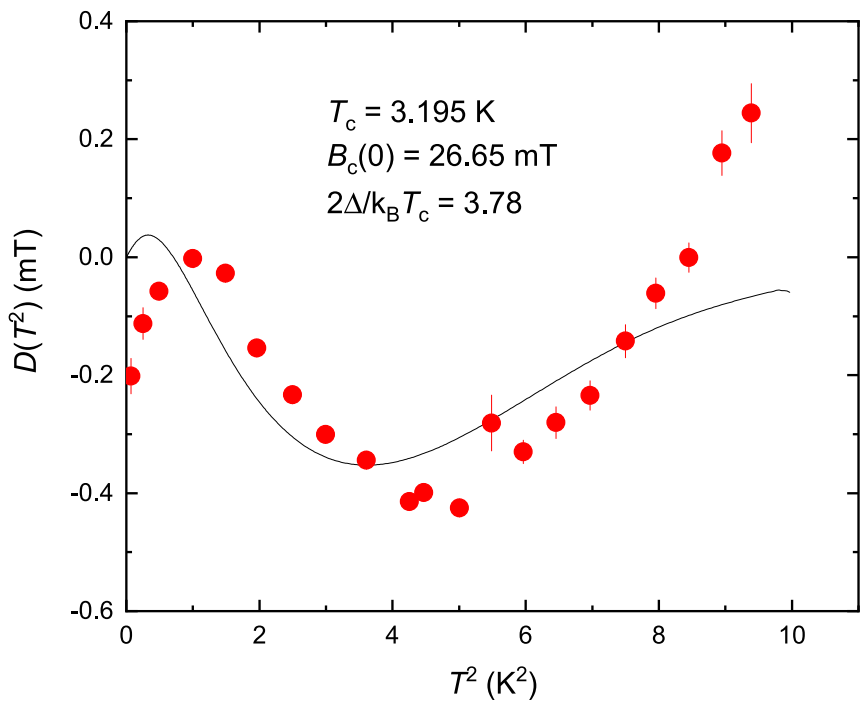

FIG. 6. (a) The deviation function $D\left(T^{2}\right)=B_{\mathrm{c}}(T)-B_{\mathrm{c}}(0)[1-$ $\left.\left(T / T_{\mathrm{c}}\right)^{2}\right]$ obtained within the framework of the $\alpha$ model. The points are the experimental data. 
[1] B. T. Matthias, J. Phys. Chem. Solids 10, 342 (1959).

[2] J. Bardeen, L. N. Cooper, and J. R. Schrieffer, Phys. Rev. 106, 162 (1957).

[3] C. Poole, H. Farach, R. Creswick, and R. Prozorov, Superconductivity, 3rd ed. (Elsevier, Amsterdam, 2014).

[4] D. Rebar, Ph.D. dissertation, Louisiana State University, 2015.

[5] A. Amon, E. Svanidze, R. Cardoso-Gil, M. N. Wilson, H. Rosner, M. Bobnar, W. Schnelle, J. W. Lynn, R. Gumeniuk, C. Hennig, G. M. Luke, H. Borrmann, A. Leithe-Jasper, and Yu. Grin, Phys. Rev. B 97, 014501 (2018).

[6] D. J. Rebar, S. M. Birnbaum, J. Singleton, M. Khan, J. C. Ball, P. W. Adams, J. Y. Chan, D. P. Young, D. A. Browne, and J. F. Di Tusa, Phys. Rev. B 99, 094517 (2019).

[7] D. Singh, A. D. Hillier, and R. P. Singh, Phys. Rev. B 99, 134509 (2019).

[8] J. Beare, M. Nugent, M. N. Wilson, Y. Cai, T. J. S. Munsie, A. Amon, A. Leithe-Jasper, Z. Gong, S. L. Guo, Z. Guguchia, Y. Grin, Y. J. Uemura, E. Svanidze, and G. M. Luke, Phys. Rev. B 99, 134510 (2019).

[9] E. Bauer, G. Hilscher, H. Michor, Ch. Paul, E. W. Scheidt, A. Gribanov, Yu. Seropegin, H. Nöel, M. Sigrist, and P. Rogl, Phy. Rev. Lett. 92, 027003 (2004).

[10] R. P. Kaur, D. F. Agterberg, and M. Sigrist, Phys. Rev. Lett. 94, 137002 (2005).

[11] H. Q. Yuan, D. F. Agterberg, N. Hayashi, P. Badica, D. Vandervelde, K. Togano, M. Sigrist, and M. B. Salamon, Phys. Rev. Lett. 97, 017006 (2006).

[12] R. Khasanov, I. L. Landau, C. Baines, F. La Mattina, A. Maisuradze, K. Togano, and H. Keller, Phys. Rev. B 73, 214528 (2006).

[13] A. P. Schnyder, S. Ryu, A. Furusaki, and A. W. W. Ludwig, Phys. Rev. B 78, 195125 (2008).

[14] A. Maisuradze, M. Nicklas, R. Gumeniuk, C. Baines, W. Schnelle, H. Rosner, A. Leithe-Jasper, Yu. Grin, and R. Khasanov, Phys. Rev. Lett. 103, 147002 (2009).

[15] P. S. Häfliger, R. Khasanov, R. Lortz, A. Petrović, K. Togano, C. Baines, B. Graneli, and H. Keller, J. Supercond. Nov. Magn. 22, 337 (2009).

[16] A. Maisuradze, W. Schnelle, R. Khasanov, R. Gumeniuk, M. Nicklas, H. Rosner, A. Leithe-Jasper, Yu. Grin, A. Amato, and P. Thalmeier, Phys. Rev. B 82, 024524 (2010).

[17] B. Bradlyn, J. Cano, Z. Wang, M. G. Vergniory, C. Felser, R. J. Cava, and A. Bernevig, Science 353, aaf5037 (2016).

[18] P. Tang, Q. Zhou, and S.-C. Zhang, Phys. Rev. Lett. 119, 206402 (2017).

[19] G. Chang, S.-Y. Xu, B. J. Wieder, D. S. Sanchez, S.-M. Huang, I. Belopolski, T.-R. Chang, S. Zhang, A. Bansil, H. Lin, and M. Z. Hasan, Phys. Rev. Lett. 119, 206401 (2017).

[20] A. Tonomura, Y. Xiuzhen, K. Yanagisawa, T. Matsuda, Y. Onose, N. Kanazawa, H. Park, and Y. Tokura, Nano Lett. 12, 1673 (2012).

[21] X. Yu, Y. Onose, N. Kanazawa, J. Park, J. Han, Y. Matsui, N. Nagaosa, and Y. Tokura, Nature (London) 465, 901 (2010).

[22] X. Yu, N. Kanazawa, Y. Onose, K. Kimoto, W. Zhang, S. Ishiwata, Y. Matsui, and Y. Tokura, Nat. Mater. 10, 106 (2011).

[23] N. Kanazawa, J.-H. Kim, D. S. Inosov, J. S. White, N. Egetenmeyer, J. L. Gavilano, S. Ishiwata, Y. Onose, T. Arima, B. Keimer, and Y. Tokura, Phys. Rev. B 86, 134425 (2012).

[24] S. Seki, X. Yu, S. Ishiwata, and Y. Tokura, Science 336, 198 (2012).
[25] U. Krägeloh, Phys. Lett. A 28, 657 (1969).

[26] J. Auer and H. Ullmaier, Phys. Rev. B 7, 136 (1973).

[27] F. Bouquet, R. A. Fisher, N. E. Phillips, D. G. Hinks, and J. D. Jorgensen, Phys. Rev. Lett. 87, 047001 (2001).

[28] S. Tsuda, T. Yokoya, T. Kiss, Y. Takano, K. Togano, H. Kito, H. Ihara, and S. Shin, Phys. Rev. Lett. 87, 177006 (2001).

[29] X. K. Chen, M. J. Konstantinović, J. C. Irwin, D. D. Lawrie, and J. P. Franck, Phys. Rev. Lett. 87, 157002 (2001).

[30] P. Szabó, P. Samuely, J. Kačmarčík, T. Klein, J. Marcus, D. Fruchart, S. Miraglia, C. Marcenat, and A. G. M. Jansen, Phys. Rev. Lett. 87, 137005 (2001).

[31] R. S. Gonnelli, D. Daghero, G. A. Ummarino, V. A. Stepanov, J. Jun, S. M. Kazakov, and J. Karpinski, Phys. Rev. Lett. 89, 247004 (2002).

[32] R. Khasanov, A. Shengelaya, A. Maisuradze, F. La Mattina, A. Bussmann-Holder, H. Keller, and K. A. Müller, Phys. Rev. Lett. 98, 057007 (2007)

[33] R. Khasanov, S. Strässle, D. Di Castro, T. Masui, S. Miyasaka, S. Tajima, A. Bussmann-Holder, and H. Keller, Phys. Rev. Lett. 99, 237601 (2007).

[34] R. Khasanov, A. Shengelaya, J. Karpinski, A. BussmannHolder, H. Keller, and K. A. Müller, J. Supercond. Nov. Magn. 21, 81 (2008).

[35] S. Ideta, K. Takashima, M. Hashimoto, T. Yoshida, A. Fujimori, H. Anzai, T. Fujita, Y. Nakashima, A. Ino, M. Arita, H. Namatame, M. Taniguchi, K. Ono, M. Kubota, D. H. Lu, Z.-X. Shen, K. M. Kojima, and S. Uchida, Phys. Rev. Lett. 104, 227001 (2010).

[36] S. Kunisada, S. Adachi, S. Sakai, N. Sasaki, M. Nakayama, S. Akebi, K. Kuroda, T. Sasagawa, T. Watanabe, S. Shin, and T. Kondo, Phys. Rev. Lett. 119, 217001 (2017).

[37] H. Ding, P. Richard, K. Nakayama, K. Sugawara, T. Arakane, Y. Sekiba, A. Takayama, S. Souma, T. Sato, and T. Takahashi, Europhys. Lett. 83, 47001 (2008).

[38] D. V. Evtushinsky, D. S. Inosov, V. B. Zabolotnyy, M. S. Viazovska, R. Khasanov, A. Amato, H.-H. Klauss, H. Luetkens, Ch. Niedermayer, G. L. Sun, V. Hinkov, C. T. Lin, A. Varykhalov, A. Koitzsch, M. Knupfer, B. Büchner, A. A. Kordyuk, and S. V. Borisenko, New J. Phys. 11, 055069 (2009).

[39] R. Khasanov, D. V. Evtushinsky, A. Amato, H.-H. Klauss, H. Luetkens, Ch. Niedermayer, B. Büchner, G. L. Sun, C. T. Lin, J. T. Park, D. S. Inosov, and V. Hinkov, Phys. Rev. Lett. 102, 187005 (2009).

[40] R. Khasanov, A. Maisuradze, H. Maeter, A. Kwadrin, H. Luetkens, A. Amato, W. Schnelle, H. Rosner, A. Leithe-Jasper, and H.-H. Klauss, Phys. Rev. Lett. 103, 067010 (2009).

[41] R. Khasanov, A. Amato, P. K. Biswas, H. Luetkens, N. D. Zhigadlo, and B. Batlogg, Phys. Rev. B 90, 140507(R) (2014).

[42] R. Khasanov, W. R. Meier, S. L. Bud'ko, H. Luetkens, P. C. Canfield, and A. Amato, Phys. Rev. B 99, 140507(R) (2019).

[43] M. Ruby, B. W. Heinrich, J. I. Pascual, and K. J. Franke, Phys. Rev. Lett. 114, 157001 (2015).

[44] J. Singh, A. Jayaraj, D. Srivastava, S. Gayen, A. Thamizhavel, and Y. Singh, Phys. Rev. B 97, 054506 (2018).

[45] A. Amato, H. Luetkens, K. Sedlak, A. Stoykov, R. Scheuermann, M. Elender, A. Raselli, and D. Graf, Rev. Sci. Instrum. 88, 093301 (2017).

[46] M. Tinkham, Introduction to Superconductivity (Krieger, Malabar, FL, 1975). 
[47] P. G. de Gennes, Superconductivity of Metals and Alloys (Benjamin, New York, 1966).

[48] C. Kittel, Introduction to Solid State Physics, 7th ed. (Wiley, Hoboken, NJ, 2007).

[49] R. Prozorov, Phys. Rev. Lett. 98, 257001 (2007).

[50] R. Prozorov, A. F. Fidler, J. R. Hoberg, and P. C. Canfield, Nat. Phys. 4, 327 (2008).

[51] R. Khasanov, M. M. Radonjić, H. Luetkens, E. Morenzoni, G. Simutis, S. Schönecker, W. H. Appelt, A. Östlin, L. Chioncel, and A. Amato, Phys. Rev. B 99, 174506 (2019).

[52] R. Karl, F. Burri, A. Amato, M. Donegà, S. Gvasaliya, H. Luetkens, E. Morenzoni, and R. Khasanov, Phys. Rev. B 99, 184515 (2019).

[53] R. Khasanov, H. Luetkens, A. Amato, and E. Morenzoni, Phys. Rev. B 101, 054504 (2020).

[54] H. Padamsee, J. E. Neighbor, and C. A. Shiffman, J. Low Temp. Phys. 12, 387 (1973).

[55] D. C. Johnston, Supercond. Sci. Technol. 26, 115011 (2013).

[56] A. Bussmann-Holder, R. Micnas, and A. R. Bishop, Eur. Phys. J. B. 37, 345 (2004).

[57] A. Bussmann-Holder, arXiv:0909.3603.
[58] R. Khasanov, M. Bendele, A. Amato, K. Conder, H. Keller, H.H. Klauss, H. Luetkens, and E. Pomjakushina, Phys. Rev. Lett. 104, 087004 (2010).

[59] https://www.mathcad.com.

[60] B. Mühlschlegel, Z. Phys. 155, 313 (1959).

[61] V. G. Kogan, C. Martin, and R. Prozorov, Phys. Rev. B 80, 014507 (2009).

[62] R. Gupta, A. Maisuradze, N. D. Zhigadlo, H. Luetkens, A. Amato, and R. Khasanov, Front. Phys. 8, 2 (2020).

[63] H. Kim, K. Cho, M. A. Tanatar, V. Taufour, S. K. Kim, S. L. Bud'ko, P. C. Canfield, V. G. Kogan, and R. Prozorov, Symmetry 11, 1012 (2019).

[64] Y. Bang, H.-Y. Choi, and H. Won, Phys. Rev. B 79, 054529 (2009).

[65] T. V. Trevisan, M. Schütt, and R. M. Fernandes, Phys. Rev. B 98, 094514 (2018).

[66] A. Suter and B. M. Wojek, Phys. Procedia 30, 69 (2012).

[67] R. Prozorov and V. G. Kogan, Phys. Rev. Appl. 10, 014030 (2018).

[68] A. Yaouanc and P. Dalmas de Réotier, Muon Spin Rotation, Relaxation and Resonance: Applications to Condensed Matter (Oxford University Press, Oxford, 2011). 